\title{
ROBUST OUTPUT REGULATION OF UNCERTAIN CHAOTIC SYSTEMS WITH INPUT MAGNITUDE AND RATE CONSTRAINTS
}

\author{
Carlos M.N. VELOSA*, Kouamana BOUSSON* \\ „LAETA-UB//AeroG \& Avionics and Control Laboratory, Department of Aerospace Sciences, University of Beira Interior, 6201-001 Covilhã, Portugal \\ carlosvelosaeng@hotmail.com, k1bousson@yahoo.com
}

received 12 December 2014, revised 18 December 2015, accepted 18 December 2015

\begin{abstract}
The problem of output regulation deserves a special attention particularly when it comes to the regulation of nonlinear systems. It is well-known that the problem is not always solvable even for linear systems and the fact that some demanding applications require not only magnitude but also rate actuator constraints makes the problem even more challenging. In addition, real physical systems might have parameters whose values can be known only with a specified accuracy and these uncertainties must also be considered to ensure robustness and on the other hand because they can be crucial for the type of behaviour exhibited by the system as it happens with the celebrated chaotic systems. The present paper proposes a robust control method for output regulation of chaotic systems with parameter uncertainties and subjected to magnitude and rate actuator constraints. The method is an extension of a work recently addressed by the same authors and consists in decomposing the nonlinear system into a stabilizable linear part plus a nonlinear part and in finding a control law based on the small-gain principle. Numerical simulations are performed to validate the effectiveness and robustness of the method using an aeronautical application. The output regulation is successfully achieved without exceeding the input constraints and stability is assured when the parameters are within the specified intervals. Furthermore, the proposed method does not require much computational effort because all the control parameters are computed offline.
\end{abstract}

Keywords: Output Regulation, Actuator Constraints, Parametric Uncertainties, Robust Control, Chaos, Aeroelastic System

\section{INTRODUCTION}

Chaotic systems have attracted significant attention from researchers over the past twenty years and there has been since then a large effort in attempting to develop and improve control techniques for such a class of systems. Chaotic systems are known to be essentially characterized by high sensitivity to initial conditions and to parameter variations, and for that reason, any effective control technique must be robust against parameter uncertainties. On the other hand, most of real-world applications require a control not only with input magnitude constraints but also with rate constraints due to electrical, mechanical and other physical limitations.

Pecora and Carroll (1990) and Ott et al. (1990) were the pioneers in introducing techniques for the control and synchronization specifically of chaotic systems. Nevertheless, despite of their elegant strategies, actuator constraints are not considered and plant uncertainties are also not taken into account. The classical $\mathrm{H}_{\infty}$ control introduced by Zames (1981) became very popular precisely because it considers, among others, parameter uncertainties. The Quantitative Feedback Theory (QFT) developed by Horowitz (2001) is another robust technique that prevails nowadays and is quite effective for systems with parametric uncertainties. However, robust control techniques have limitations when used to control systems subject to actuator constraints. Mathematical manipulations can be obviously carried out to introduce such constraints, but its implementation requires an appropriate data structure and some precautions should be taken into account to prevent windup phenomena, (Galeani et al., 2007; Hippe, 2006). Advanced control techniques based on Linear Matrix Ine- qualities (LMI's) are effective and allow to include, among others, plant uncertainties and actuator constraints, (Deng and Xu, 2010; Tanaka and Wang, 2001). Meanwhile, dealing with LMI-based controller design requires modelling experience with positive definite matrices because some parameters should be relaxed in order to formulate a subsequent convex optimization problem that is not too conservative. It may occur that the control saturates while the output is still too far from the setpoint, which may lead to the instability of the closed-loop system. Bousson and Velosa (2014), Velosa and Bousson $(2014,2015)$ have recently tackled the problem of control and synchronization of chaotic systems with actuator constraints. But, despite of robustness against external disturbances and also to parameter uncertainties, their techniques do not consider interval-valued parameter uncertainties.

The present paper aims at proposing a generalized robust method for output regulation of nonlinear complex systems that takes into account input magnitude and rate constraints and deals explicitly with parametric uncertainties. The work is motivated by the fact that some highly demanding applications such as aerospace and aeronautical systems require this type of constraints and because in most cases the parameters of the system can be only known with a certain accuracy.

The paper is structured as follows: the problem to be solved is stated in section 2; section 3 presents a solution to problem, which is an extension of the work recently proposed by Velosa and Bousson (2015) based on the $\mathrm{H}_{\infty}$ control, more precisely, based on the small-gain principle; section 4 deals with numerical simulations to validate the effectiveness and robustness of the method coping with suppressing the undesired oscillations of an 
aeroelastic system; and section 5 discusses about the simulation results with concluding remarks.

\section{PROBLEM STATEMENT}

Consider the problem of robust output regulation of a continuous time-invariant nonlinear system with parametric uncertainties described as (1):

$\dot{x}=f(x, \theta)+B(\theta) u$

$y=C(\theta) x$

Controlled system

$\dot{x}_{r}=f_{r}\left(x_{r}\right)$

$y_{r}=C_{r} x_{r}$

Reference system

where the purpose is to find an admissible control subject to magnitude and rate constraints such that its outputs follow insofar as possible reference signals generated by a given system (2).

In equations (1) and (2), $x \in \mathbb{R}^{n}, x_{r} \in \mathbb{R}^{n_{r}}$ denote the state vectors, $y, y_{r} \in \mathbb{R}^{p}$ the output vectors, $u \in \mathbb{R}^{m}$ the control vector, $\theta \in \mathbb{R}^{q}$ the vector of parameters which is known only with a certain degree of accuracy, $C(\theta) \in \mathbb{R}^{p \times n}, C_{r} \in \mathbb{R}^{p \times n_{r}}$ the output matrices, $B(\theta) \in \mathbb{R}^{n \times m}$ the control matrix, $f, f_{r}$ two smooth nonlinear functions, and $\dot{x}=d x / d t$.

Writing the problem with the mathematical formalities, the objective summarizes to the following:

Problem to be solved: Find a control $u(t)$ such that conditions (3) and (4) hold for any $t \geq \tau, \tau>t_{0}$, with knowledge that the parameters of (1) may take any values within the limits specified by (5):

$\left\|e_{y}\right\|=\left\|y(t)-y_{r}(t)\right\| \leq \varepsilon$

$\|u(t)\|_{\infty} \leq \eta \quad, \quad\|\dot{u}(t)\|_{\infty} \leq v$

$\theta_{i} \in\left[\theta_{i, \min }, \theta_{i, \max }\right], \quad i=1, \ldots, q$

In inequality (3), \|. $\|$ denotes an appropriate norm and $\varepsilon \geq 0$ should be as small as possible for a proper regulation. Parameters $\eta \geq 0$ and $v \geq 0$ denote respectively the magnitude and rate constraints of the actuators dynamics.

\section{THE PROPOSED METHOD}

Consider a continuous-time, time-invariant linear system given as follows:

$\dot{x}=A\left(\theta_{0}\right) x+B_{1}\left(\theta_{0}\right) \sigma_{\eta, v}(u)+B_{2} w+B_{3} d$

$\dot{w}=S w+r$

$y=C_{1}\left(\theta_{0}\right) x+D_{13} d$

$e=C_{1}\left(\theta_{0}\right) x+D_{12} w$

$z=C_{2} x+D_{21} \sigma_{\eta, v}(u)$

where the first equation describes a plant, with state $x \in \mathbb{R}^{n}$, input $u \in \mathbb{R}^{m}$, and subject to the effect of external disturbances and parametric uncertainties represented respectively by $B_{2} W$ and $B_{3} d$. Vector $\theta_{0}$ denotes the nominal value of parameter vector $\theta$. The third equation refers to the real plant output $y \in$ $\mathbb{R}^{p}$, which is also affected by parametric uncertainties represented by $D_{13} d$. The fourth equation defines the error $e \in \mathbb{R}^{p}$ between the plant output $C_{1}\left(\theta_{0}\right) x$ and the reference signal $-D_{12} \mathrm{~W}$ which the plant output is required to track. The second equation describes an exosystem, with state $w \in \mathbb{R}^{s}$, that models simulta- neously the class of plant disturbances and the reference signals taken into consideration. The last equation, $z \in \mathbb{R}^{n+m}$, represents the performance outputs which are intended to be influenced in a desired way.

In the sequel, the notations $A\left(\theta_{0}\right) \equiv A_{0}, B_{1}\left(\theta_{0}\right) \equiv B_{1,0}$ and $C_{1}\left(\theta_{0}\right) \equiv C_{1,0}$ stand for the corresponding matrices with nominal parameter vector $\theta_{0}$ taken equal to the midpoint of the parameter box in equation (5).

Let the control constraints as presented in (4) be enforced by a functional differential operator of the form $\sigma_{\eta, v}(u)=$ $\lim _{\mu \rightarrow \infty} x_{\mu}$, with $x_{\mu}$ described as:

$\dot{x}_{\mu}=\sigma_{v}\left(\mu\left(\sigma_{\eta}(u)-x_{\mu}\right)\right)$

$x_{\mu}\left(t_{0}\right)=\sigma_{\eta}\left(u\left(t_{0}\right)\right)$

where $\sigma_{h}($.$) denotes a saturation function of limit h$, defined by:

$\sigma_{h}(s)=\operatorname{sgn}(s) \cdot \min \{|s|, h\}$

The parametric uncertainties of system (6) can be expressed as a feedback of the performance output through an appropriate selection of matrices $C_{2}$ and $D_{21}$. That is, $d=\Delta z$, with:

$\Delta=\left[\begin{array}{cc}\Delta A & \Delta B_{1} \\ \Delta C_{1} & 0_{p \times m}\end{array}\right]$

where:

$\|\Delta A\|=\left\|A(\theta)-A\left(\theta_{0}\right)\right\| \leq a$
$\left\|\Delta B_{1}\right\|=\left\|B_{1}(\theta)-B_{1}\left(\theta_{0}\right)\right\| \leq b$
$\left\|\Delta C_{1}\right\|=\left\|C_{1}(\theta)-C_{1}\left(\theta_{0}\right)\right\| \leq c$

and it can be easily shown that $\|\Delta\|_{\infty} \leq 1 / \gamma, \gamma>0$, where $\gamma$ is computed according to the expression, (Bernhard, 2002):

$\gamma=\frac{1}{\sqrt{\frac{a^{2}+b^{2}+c^{2}+\sqrt{\left(a^{2}+b^{2}+c^{2}\right)^{2}-(2 b c)^{2}}}{2}}}$

Theorem 1: The problem of robust output regulation via statefeedback for the system (6) is solvable if the following conditions are true, (Bernhard, 2002; Saberi et al., 2011):

i. The pair $\left(A_{0}, B_{1,0}\right)$ is controllable and $A_{0}$ has all its eigenvalues in the closed left-half plane.

ii. $D_{21}^{T} D_{21}$ is invertible and $D_{21}^{T} C_{2}=0$.

iii. The pair $\left(C_{2}, A_{0}\right)$ has no unobservable modes on the imaginary axis.

iv. There exist two matrices $\Pi$ and $\Gamma$ such that:

a) they solve the regulator equations:

$$
\begin{aligned}
& \Pi S=A_{0} \Pi+B_{1,0} \Gamma+B_{2} \\
& 0=C_{1,0} \Pi+D_{12}
\end{aligned}
$$

b) for a given $\xi>0$ and $T \geq 0$ they satisfy the following inequalities:

$$
\left\|\Gamma w+B_{1,0}^{+} \Pi r\right\|_{\infty, T} \leq \eta-\xi
$$

and: $\left\|\Gamma S w+\Gamma r+B_{1,0}^{+} \Pi \dot{r}\right\|_{\infty, T} \leq v-\xi$.

Under these conditions, the family of linear static statefeedback laws given by:

$u=-B_{1,0}^{T} P x+\left(B_{1,0}^{T} P \Pi+\Gamma\right) w+B_{1,0}^{+} \Pi r$ 
where $B_{1,0}^{+}$denotes the pseudo-inverse matrix of $B_{1,0}$ and $P$ is a symmetric and positive semi-definite solution, if there exists, of the following algebraic Riccati equation:

$A_{0}^{T} P+P A_{0}-P B_{1,0} B_{1,0}^{T} P+\gamma^{-2} P B_{3} B_{3}^{T} P+C_{2}^{T} C_{2}=0$

such that the matrix $A_{0}-B_{1,0} B_{1,0}^{T} P+\gamma^{-2} B_{3} B_{3}^{T} P$ is stable, i.e., all its eigenvalues have negative real parts, steers the output error $e(t)$ of system (6) to the origin when $t \rightarrow \infty$, $\lim _{t \rightarrow \infty} e(t)=0$, and guarantees that the closed-loop transfer matrix from $d$ to $z$ satisfies $\left\|T_{z, d}\right\|_{\infty}<\gamma$ due to the small-gain theorem, (Zames, 1981).

A way to solve the regulator equations (12) and the Riccati equation (14) is presented in appendices (1) and (2) respectively.

Let matrices $B_{2}, B_{3}, C_{2}, D_{13}, D_{12}, D_{21}$, be given as:

$B_{2}=\left[\begin{array}{ll}I_{n} & 0_{n \times n_{r}}\end{array}\right] \quad, \quad B_{3}=\left[\begin{array}{ll}I_{n} & 0_{n \times p}\end{array}\right]$

$D_{13}=\left[\begin{array}{ll}0_{p \times n} & I_{p}\end{array}\right] \quad, \quad D_{12}=\left[0_{p \times n}-C_{r}\right]$

$D_{21}=\left[\begin{array}{c}0_{n \times m} \\ I_{m}\end{array}\right] \quad, \quad C_{2}=\left[\begin{array}{c}I_{n} \\ 0_{m \times n}\end{array}\right]$

Decompose now the nonlinear functions of systems (1) and (2) into the form of a linear part plus a nonlinear part so that the linear part of (1) is stable for the nominal parameters, that is:

$f\left(x, \theta_{0}\right)=A_{0} x+\varphi\left(x, \theta_{0}\right)$

$f_{r}\left(x_{r}\right)=A_{r} x_{r}+\varphi_{r}\left(x_{r}\right)$

$A_{0}=\left.\frac{\partial f}{\partial x}\right|_{x=x^{*}} \quad, \quad A_{r}=\left.\frac{\partial f_{r}}{\partial x_{r}}\right|_{x_{r}=x_{r}^{*}}$

with $x^{*}$ chosen such that:

$\operatorname{Re}\left[\lambda_{i}\left(A_{0}\right)\right] \leq 0, \quad i=1, \ldots, n$

and $\varphi$ and $\varphi_{r}$ written as:

$\varphi\left(x, \theta_{0}\right)=f\left(x, \theta_{0}\right)-A_{0} x$

$\varphi_{r}\left(x_{r}\right)=f_{r}\left(x_{r}\right)-A_{r} x_{r}$

Note that the first equation of (6) describes a perturbed linear system: in the absence of uncertainties, it represents the nominal model; and in the presence of uncertainties it represents all possible models better than the 'worst' model taken into consideration, i.e., the model for the maximum allowable uncertainty. Since the controlled system is described by a nonlinear function $f(x, \theta)=$ $A(\theta) x+\varphi(x, \theta)$, when written into the form of a nominal model plus the remaining uncertainty terms, it would be $f(x, \theta)=$ $A_{0} x+\varphi_{0}(x)+\Delta A x+\Delta \varphi(x)$. However, certain systems, and, in particular most of the chaotic systems, have their parameters only in the linear terms of the model, and under this condition the previous expression simplifies to $f(x, \theta)=A_{0} x+\varphi(x)+$ $\Delta A x$. Lorenz, Chen, Lu systems are examples of well-known chaotic systems for which all the parameters appear exclusively in the linear terms. For such systems, the uncertainty $\Delta A x$ can be easily characterized by the term $B_{3} d$ according to (9).

The arrangement above allows one to write the exosystem of (6) in its exact form $\dot{w}=S w+r$, generating thus the nonlinear part of the controlled system, $\varphi \equiv \varphi\left(x, \theta_{0}\right)$, and the reference state vector, $x_{r}$ :

$\left[\begin{array}{c}\dot{\varphi} \\ \dot{x}_{r}\end{array}\right]=\left[\begin{array}{cc}0_{n \times n} & 0_{n \times n_{r}} \\ 0_{n_{r} \times n} & A_{r}\end{array}\right]\left[\begin{array}{c}\varphi \\ x_{r}\end{array}\right]+\left[\begin{array}{c}\dot{\varphi}(x) \\ \varphi_{r}\left(x_{r}\right)\end{array}\right]$ where:

$\dot{\varphi}(x)=\left(\frac{\partial f\left(x, \theta_{0}\right)}{\partial x}-A_{0}\right) f\left(x, \theta_{0}\right)$

\section{SIMULATION RESULTS}

In this section numerical simulations are performed to validate the proposed approach. The model under consideration is an aeroelastic system with two degrees-of-freedom (Fig. 1), and the purpose is to suppress limit cycle oscillations (flutter) and eventual chaotic motion that can lead to structural failures by material fatigue.

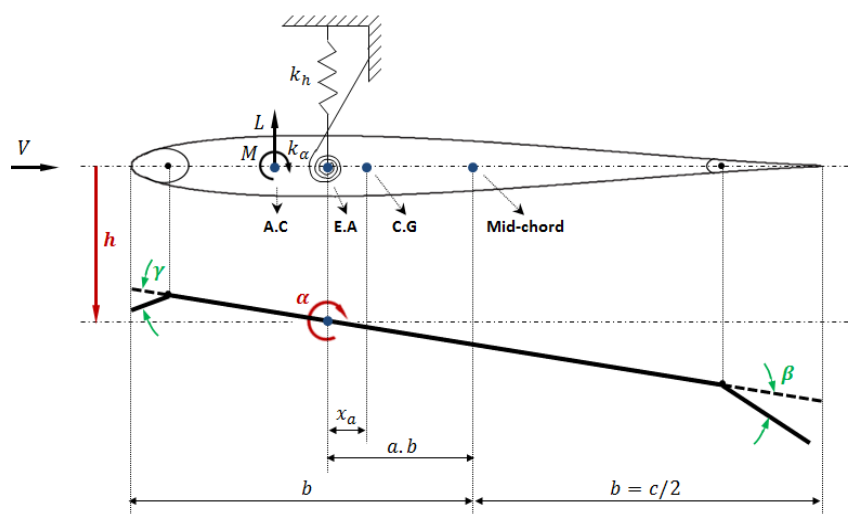

Fig. 1. Aeroelastic system with two degrees-of-freedom

The mathematical equations of motion for an aeroelastic system with two degrees-of-freedom as described in (Fig. 1) have been established in many references, (Alstrom et al., 2010; Chen et al., 2012; Demenkov, 2008; Vikhorev et al., 2008; Wang et al., 2013). Following (Demenkov, 2008), the dynamical model can be expressed, denoting the state vector by $x=\left[\begin{array}{llll}h & \alpha & \dot{h} & \dot{\alpha}\end{array}\right]^{T}$, the output vector by $y=\left[\begin{array}{ll}h & \alpha\end{array}\right]^{T}$ and the control vector by $u=$ $\left[\begin{array}{ll}\beta & \gamma\end{array}\right]^{T}$, as:

$\dot{x}=f(x)+B u$

$y=C x$

with:

$f(x)=\left[\begin{array}{cc}0_{2} & I_{2} \\ -F^{-1} C_{\alpha}(\alpha) & -F^{-1} G\end{array}\right] x$

$B=\left[\begin{array}{c}0_{2} \\ -F^{-1} L\end{array}\right], \quad C=\left[\begin{array}{ll}I_{2} & 0_{2}\end{array}\right]$

wherein:

$F=\left[\begin{array}{cc}m_{T} & m_{W} x_{\alpha} b \\ m_{W} x_{\alpha} b & I_{\alpha}\end{array}\right]$

$L=\left[\begin{array}{cc}-\rho V^{2} b s C_{l \beta} & -\rho V^{2} b s C_{l \gamma} \\ \rho V^{2} b^{2} s C_{m \beta-e f f} & \rho V^{2} b^{2} s C_{m \gamma-e f f}\end{array}\right]$

$G=\left[\begin{array}{cc}c_{h}+\rho V b s C_{l \alpha} & \rho V b^{2} s C_{l \alpha}(1 / 2-a) \\ -\rho V b^{2} s C_{m \alpha-e f f} & c_{a}-\rho V b^{3} s C_{m \alpha-e f f}(1 / 2-a)\end{array}\right]$

$C_{\alpha}(\alpha)=\left[\begin{array}{cc}k_{h} & \rho V^{2} b s C_{l \alpha} \\ 0 & k_{\alpha}(\alpha)-\rho V^{2} b^{2} s C_{m \alpha-e f f}\end{array}\right]$

and: 
$k_{\alpha}(\alpha)=12.77+53.47 \alpha+1003 \alpha^{2}$

$x_{\alpha}=-(0.0998+a) \quad, \quad r_{c g}=b x_{\alpha}$

$I_{\alpha}=I_{c a m}+I_{c g-\text { wing }}+m_{\text {wing }} r_{c g}^{2}$

$C_{m \alpha-e f f}=(1 / 2+a) C_{l \alpha}+2 C_{m \alpha}$

$C_{m \beta-e f f}=(1 / 2+a) C_{l \beta}+2 C_{m \beta}$

$C_{m \gamma-e f f}=(1 / 2+a) C_{l \gamma}+2 C_{m \gamma}$

The plunge displacement $h$ and angles $\alpha, \beta, \gamma$ are expressed respectively in meters and radians. The parameters of the system are given in Tab. 1. One considers that the stability and control derivatives are estimated; hence they are known only with a certain degree of accuracy. Let the uncertainty be $10 \%$ around the nominal values $\theta_{0}$, i.e., $\theta \in\left[0.90 \theta_{0}, 1.10 \theta_{0}\right]$ where $\theta=$ $\left[C_{l \alpha}, C_{m \alpha}, C_{l \beta}, C_{m \beta}, C_{l \gamma}, C_{m \gamma}\right]^{T}$.

Tab. 1. Parameters of the aeroelastic system.

\begin{tabular}{|l|l|c|}
\hline Parameter & Value & Tolerance \\
\hline$\rho$ & $1.225 \mathrm{~kg} / \mathrm{m}^{3}$ & - \\
\hline$a$ & -0.6719 & - \\
\hline$b$ & $0.1905 \mathrm{~m}$ & - \\
\hline$s$ & $0.5945 \mathrm{~m}$ & - \\
\hline$k_{h}$ & $2844 \mathrm{~N} / \mathrm{m}$ & - \\
\hline$c_{h}$ & $27.43 \mathrm{~kg} / \mathrm{s}$ & - \\
\hline$C_{\alpha}$ & $0.0360 \mathrm{~kg} \cdot \mathrm{m}^{2} / \mathrm{s}$ & - \\
\hline$m_{\text {wing }}$ & $4.340 \mathrm{~kg}$ & - \\
\hline$m_{W}$ & $5.230 \mathrm{~kg}$ & - \\
\hline$m_{T}$ & $15.57 \mathrm{~kg}$ & - \\
\hline$I_{c a m}$ & $0.04697 \mathrm{~kg} \cdot \mathrm{m}^{2}$ & - \\
\hline$I_{c g-\text { wing }}$ & $0.04342 \mathrm{~kg} \cdot \mathrm{m}^{2}$ & - \\
\hline$C_{l \alpha}$ & 6.757 & $\pm 10 \%$ \\
\hline$C_{m \alpha}$ & 0 & $\pm 10 \%$ \\
\hline$C_{l \beta}$ & 3.774 & $\pm 10 \%$ \\
\hline$C_{m \beta}$ & -0.6719 & $\pm 10 \%$ \\
\hline$C_{l \gamma}$ & -0.1566 & $\pm 10 \%$ \\
\hline$C_{m \gamma}$ & -0.1005 & $\pm 10 \%$ \\
\hline
\end{tabular}

Notice that all uncertain parameters are only in linear terms of the model (21-24) and therefore the method proposed in the previous section can be perfectly applied. That is, the uncertainties reflect only in the linear terms $\Delta A x+\Delta B u$ and not in $\Delta \varphi(x)$.

Decomposing $f(x)$ as indicated in (16) considering the state $x^{*}=[0,10 . \pi / 180,0,0]^{T}$, one has that:

$A_{0}=\left[\begin{array}{cccc}0 & 0 & 1 & 0 \\ 0 & 0 & 0 & 1 \\ -214.1 & 26.2 & -2.9 & -0.2 \\ 859.9 & -1011.3 & 8.7 & -0.2\end{array}\right]$

$\lambda\left(A_{0}\right)=\left\{\begin{array}{l}-0.195+32.247 i \\ -0.195-32.247 i \\ -1.352+13.593 i \\ -1.352-13.593 i\end{array}\right\}$

$B_{1,0}=\left[\begin{array}{cc}0 & 0 \\ 0 & 0 \\ 4.225 & -0.544 \\ 49.74 & 8.013\end{array}\right]$

$\operatorname{rank}\left(\left[\begin{array}{llllll}B_{1,0} & A_{0} B_{1,0} & A_{0}^{2} B_{1,0} & \ldots & A_{0}^{n-1} B_{1,0}\end{array}\right]\right)=4=n$ and, therefore, the pair $\left(A_{0}, B_{1,0}\right)$ is controllable (first condition of theorem 1). For matrices $B_{2}, B_{3}, D_{13}, D_{12}, D_{21}, C_{2}$ as stated in (15), the second and third conditions of theorem 1 are fulfilled as well:

$D_{21}^{T} D_{21}=\left[\begin{array}{ll}0_{n \times m} & I_{m}\end{array}\right]\left[\begin{array}{c}0_{n \times m} \\ I_{m}\end{array}\right]=I_{m}=I_{m}^{-1}$

$D_{21}^{T} C_{2}=\left[\begin{array}{ll}0_{n \times m} & I_{m}\end{array}\right]\left[\begin{array}{c}I_{n} \\ 0_{m \times n}\end{array}\right]=0_{m \times n}$

$\operatorname{rank}\left(\left[\begin{array}{lllll}C_{2} & C_{2} A_{0} & C_{2} A_{0}^{2} & C_{2} A_{0}^{n-1}\end{array}\right]^{T}\right)=4=n$

Solving the regulator equations (12), yields:

$\Pi=\left[\begin{array}{rrrrrr}0 & 0 & 0 & 0 & 1 & 0 \\ 0 & 0 & 0 & 0 & 0 & 1 \\ -1 & 0 & 0 & 0 & 0 & 0 \\ 0 & -1 & 0 & 0 & 0 & 0\end{array}\right]$

$\Gamma \cong\left[\begin{array}{rrrrrr}-0.302 & -0.025 & -0.132 & -0.009 & 20.48 & 5.588 \\ 2.960 & 0.127 & 0.816 & -0.069 & -234.5 & 91.53\end{array}\right]$

Computing $\gamma$ for the maximum admissible uncertainty according to $(9-11), \theta=1.10 \theta_{0}$, yields $\gamma=0.198$, and the solution of the algebraic Riccati equation (14) is:

$P \cong\left[\begin{array}{crrr}15.38 & -14.10 & 0.163 & 0.040 \\ * & 13.67 & -0.192 & -0.005 \\ * & * & 0.019 & -0.013 \\ * & * & * & 0.013\end{array}\right]$

where two additional weighting parameters were considered to assist in finding a symmetric and positive semi-definite matrix $\left(P=P^{T}, P \geq 0\right)$ equal to $\varepsilon_{Q}=10^{-2}$ and $\varepsilon_{R}=10^{4}$, $\left(\varepsilon_{Q}, \varepsilon_{R}>0\right)$, that is, matrices $Q$ and $R$ are defined as $Q=$ $\varepsilon_{Q} \cdot Q$ and $R=\varepsilon_{R} \cdot R$ (see appendix 2 for details).

Since the control objective in this particular application is to steer the outputs to the origin, a reference system of dimension two departing from zero initial condition $x_{r}(0)=\left[\begin{array}{ll}0 & 0\end{array}\right]^{T}$ is appropriate to generate the desired outputs $y_{r}=\left[\begin{array}{ll}h & \alpha\end{array}\right]^{T}=$ $\left[\begin{array}{ll}0 & 0\end{array}\right]^{T}$ :

$\dot{x}_{r}=A_{r} x_{r}+\varphi_{r}\left(x_{r}\right)$

$y_{r}=C_{r} x_{r}$

with:

$A_{r}=\left[\begin{array}{ll}0 & 0 \\ 0 & 0\end{array}\right], \quad \varphi_{r}\left(x_{r}\right)=\left[\begin{array}{l}0 \\ 0\end{array}\right], \quad C_{r}=\left[\begin{array}{ll}1 & 0 \\ 0 & 1\end{array}\right]$

For simulation purposes, the differential equations of systems (6) and (7) were solved simultaneously through the RK-Butcher method between $t_{0}=0$ and $t_{f}=12 \mathrm{~s}$, with a step of $d t=$ $0.01 \mathrm{~s}$, and departing from initial conditions (31). In (7), $\mu$ was set to $\mu=100$. The freestream velocity was chosen as $V=$ $13.40 \mathrm{~m} / \mathrm{s}$ and corresponds to $25 \%$ above the predicted flutter velocity $V_{F} \in[10.72,10.73] \mathrm{m} / \mathrm{s}$. The control is switched on at $t_{u_{o n}}=6 \mathrm{~s}$, a time-instant at which the aeroelastic system is already in a limit cycle oscillation. The amplitudes and velocities of the control surfaces are limited to $\beta, \gamma \in[-25,25]^{\circ}$ and $\dot{\beta}, \dot{\gamma} \in[-1000,1000]^{\circ} / \mathrm{s}$, see (32). 


\section{Initial conditions:}

$x_{0}=\left[\begin{array}{llll}0.02 & 5 . \pi / 180 & 0.00 & 0 . \pi / 180\end{array}\right]^{T}$

$x_{r, 0}=\left[\begin{array}{ll}0.00 & 0 . \pi / 180\end{array}\right]^{T}$

$\varphi_{0}=f\left(x_{0}, \theta_{0}\right)-A_{0} x_{0}$

$x_{\mu, 0}=\sigma_{\eta}\left(\left[\begin{array}{ll}0 . \pi / 180 & 0 . \pi / 180\end{array}\right]^{T}\right)$

\section{Actuator constraints:}

$\|u\|_{\infty} \leq \eta=25 . \pi / 180$

$\|\dot{u}\|_{\infty} \leq v=1000 . \pi / 180$

The simulation results are depicted in Figs. 2 - 5. Figs. 2 and 3 show the time histories of the state variables $x=\left[\begin{array}{llll}h & \alpha & \dot{h} & \dot{\alpha}\end{array}\right]^{T}$, where the first two represent also the system outputs. The magnitude of the control variables, in fact, the trailing- and leading-edge

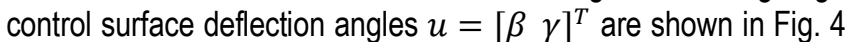
and their respective rates $\dot{u}=\left[\begin{array}{ll}\dot{\beta} & \dot{\gamma}\end{array}\right]^{T}$ in Fig. 5. The units of $h$ and $\alpha, \beta, \gamma$ are presented respectively in $\mathrm{cm}$ and degrees for a better interpretation. Fig. 6 shows $\left\|\Gamma w+B_{1,0}^{+} \Pi r\right\|_{\infty} \equiv g_{\eta}$ and $\left\|\Gamma \mathrm{S} w+\Gamma r+B_{1,0}^{+} \Pi \dot{r}\right\|_{\infty} \equiv g_{v}$ to verify (iv - b) of theorem 1.
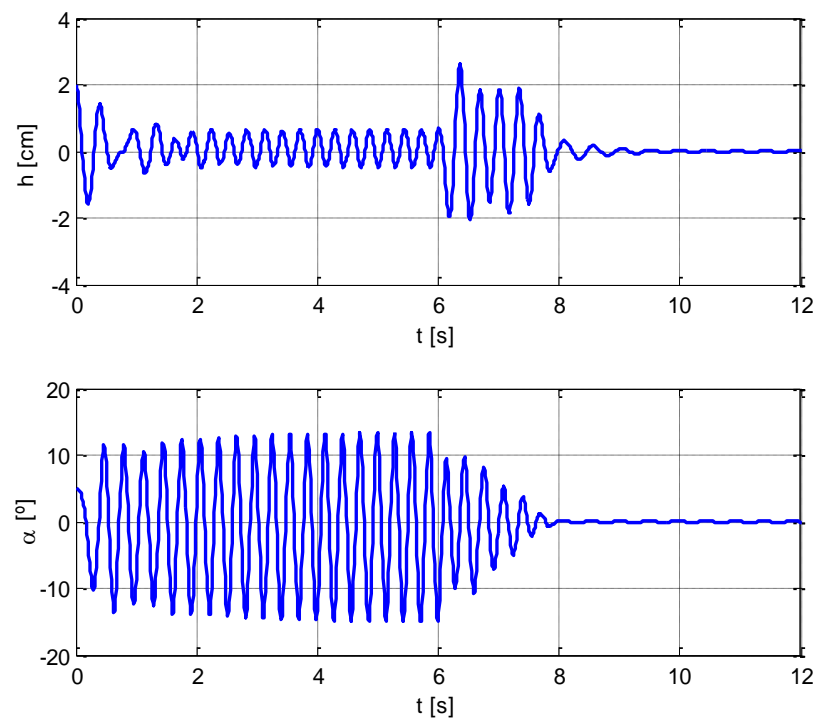

Fig. 2. State variables and outputs of the system: $h$ and $\alpha$
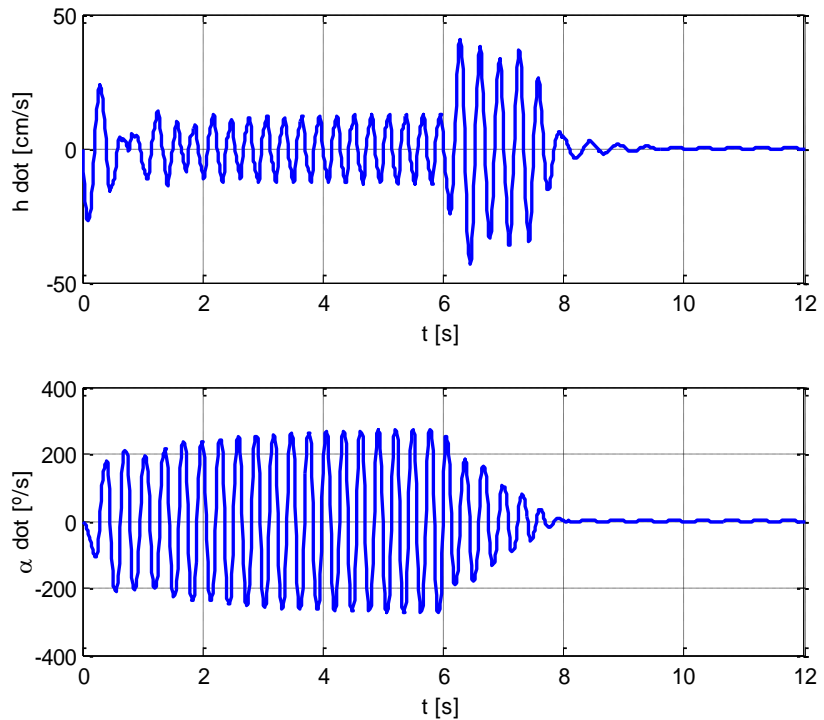

Fig. 3. State variables of the system: $\dot{h}$ and $\dot{\alpha}$
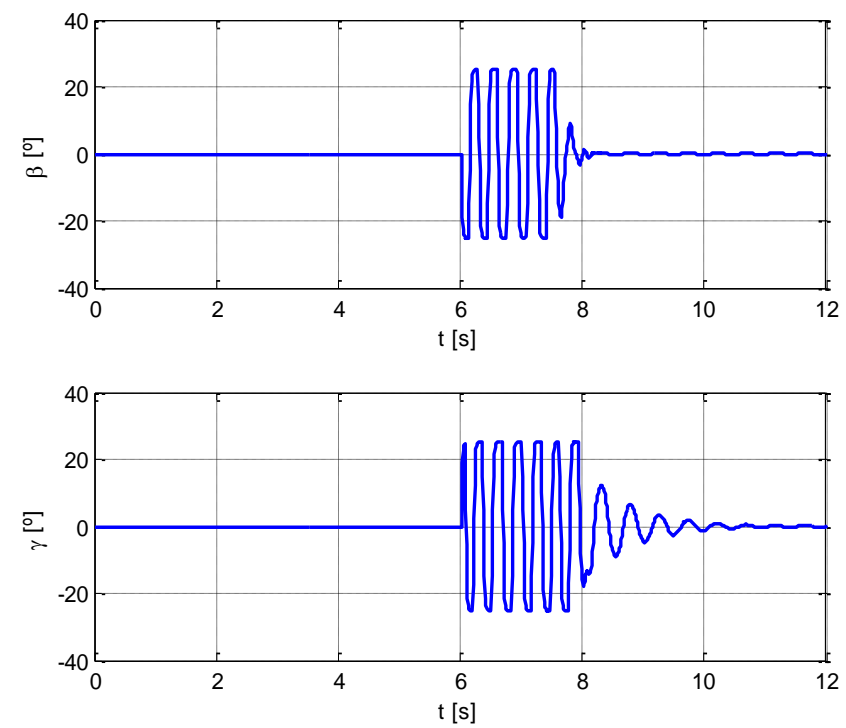

Fig. 4. Magnitude of the control variables: $\beta$ and $\gamma$. $\|u\|_{\infty} \leq 25^{\circ}$
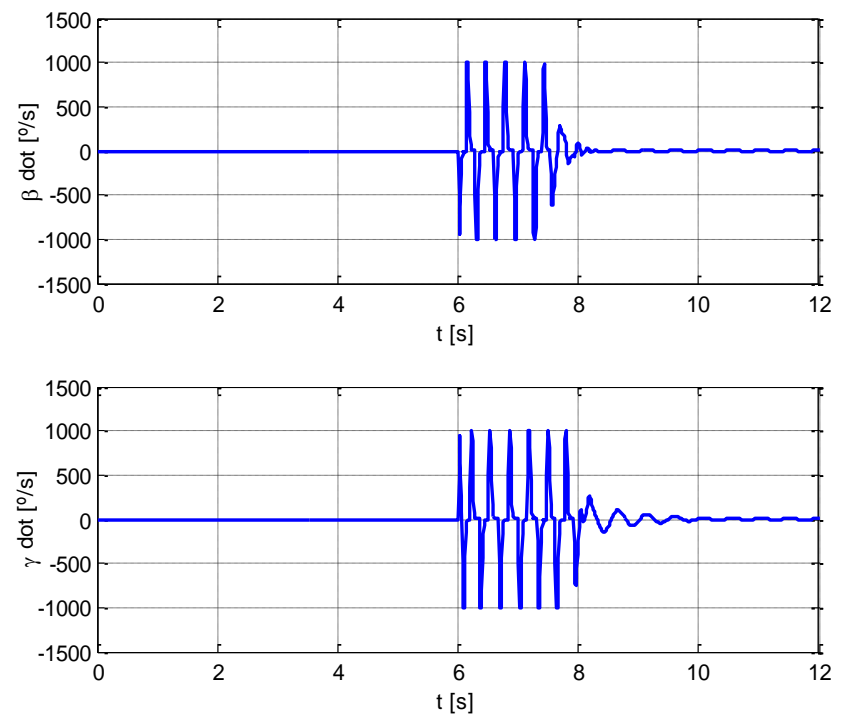

Fig. 5. Rates of the control variables: $\dot{\beta}$ and $\dot{\gamma}$. $\|\dot{u}\|_{\infty} \leq 1000 \%$ s
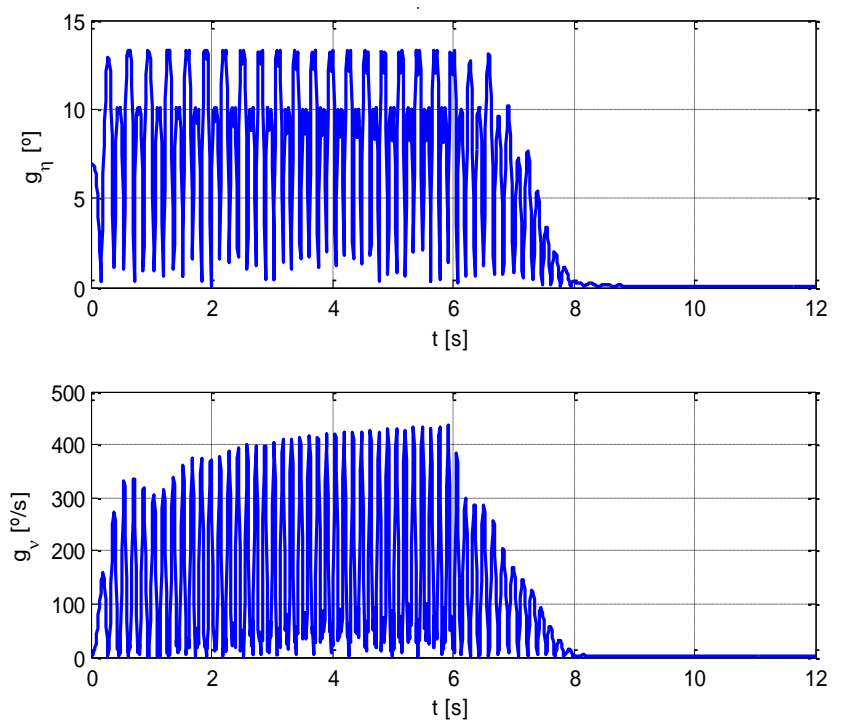

Fig. 6. $g_{\eta} \equiv\left\|\Gamma w+B_{1,0}^{+} \Pi r\right\|_{\infty} \leq 25^{\circ}$ and $g_{v} \equiv\left\|\Gamma S w+\Gamma r+B_{1,0}^{+} \Pi \dot{r}\right\|_{\infty} \leq 1000^{\circ} / \mathrm{s}$ 


\section{DISCUSSION}

The numerical simulations prove that the proposed method steers effectively the output error to the origin $\lim _{t \rightarrow \infty} e(t)=0$ assuring that the control saturation bounds are not violated, $\|u(t)\|_{\infty} \leq \eta,\|\dot{u}(t)\|_{\infty} \leq v$. Simulations were also performed for different parameter values within the specified interval $\theta \in$ $\left[0.90 \theta_{0}, 1.10 \theta_{0}\right]$, departing from different initial conditions, and for different saturation limits, $\eta$ and $v$, and in all cases the control was successfully achieved. Furthermore, since the criterion invoked to ensure robustness against parameter uncertainties is fairly strong (small-gain theorem), the control law can actually regulate the outputs even if the actual uncertainties are beyond the specified ones, or at least for slightly higher uncertainties.

Another point that deserves attention is the fact that the solution of the algebraic Riccati equation (14), $P$, should guarantee that the matrix $\tilde{A} \equiv A_{0}-B_{1,0} B_{1,0}^{T} P+\gamma^{-2} B_{3} B_{3}^{T} P$ is stable, as stated in theorem 1 , and this may restrict the maximum admissible uncertainty taken into consideration. However, a given solution $P$ is also admissible since the first two terms of $\tilde{A}$ are stable, that is, $\operatorname{Re}\left[\lambda_{i}\left(A_{0}-B_{1,0} B_{1,0}^{T} P\right)\right] \leq 0$. Simulations were carried out assuming an uncertainty of $\pm 20 \%, \theta \in\left[0.80 \theta_{0}, 1.20 \theta_{0}\right]$, condition wherein $\gamma$ is too small $(\gamma=0.099)$ and $\operatorname{Re}\left[\lambda_{i}(\tilde{A})\right] \leq$ 0 is not satisfied, and the vibrations were suppressed.

Analysing Fig. 2, one verifies that, contrarily to what happens with the angle of attack $\alpha$ where it is gradually attenuated from the time-instant at which the control is turned on, $t_{u_{o n}}=6 \mathrm{~s}$, the plunge displacement $h$ increases before starting to decrease. This means that in a critical application like the aeroelastic system presented here, some precautions should be taken into account when applying the method because the excessive increase in the plunge displacement may lead to structural failure. Nevertheless, such an undesired effect can be prevented by restricting the output, but that topic is a subject for another paper.

\section{CONCLUSION}

A robust output regulation method for chaotic systems which takes into account magnitude and rate actuator constraints has been proposed in the present paper. The method consists, in a first stage, in splitting the nonlinear model into a stable linear part and in a remaining nonlinear part. Then, the nonlinear part is generated simultaneously with the reference signals with the aid of an exosystem. Next, the uncertainties in the model are described as additional terms, taking into account the fact that most of the chaotic systems have their parameters exclusively in the linear terms. Besides, bounds are set on the magnitudes of the admissible uncertainties. In fact, these bounds are parameters that are used subsequently for the computation of an $H_{\infty}$ state feedback control law. Lastly, a differential operator is used to enforce both magnitude and rate constraints in the controlled system.

Numerical simulations are performed to validate the method using an aeroelastic system. The results show that the undesired vibrations are effectively supressed for any parameters within the specified bounds and without exceeding the actuator constraints. Furthermore, the reference signals are tracked within a rather reduced settling time taking into account that the control is subject to magnitude and rate constraints.

The control law presented in the paper is static and all its pa- rameters are computed offline. This is therefore a great asset, since the method can be easily implemented in a low-cost platform because it does not require high computational power. The fact that both magnitude and rate constraints are considered is another advantage since they are crucial for the proper operation of highly demanding applications, and many other control techniques do not consider them at all especially when parameter uncertainties are taken into consideration.

Extensions for the present work are possible, among which one emphasizes the hypothesis of considering asymmetric actuator constraints, an output feedback (instead of a full-state feedback) to take into account also measurement noise, and to extend the area of applicability of the method to systems whose parameters may also appear in the nonlinear terms.

\section{REFERENCES}

1. Alstrom, B., Marzocca, P., Bollt, E., Ahmadi, G. (2010), Controlling Chaotic Motions of a Nonlinear Aeroelastic System Using Adaptive Control Augmented with Time Delay, AIAA Guidance, Navigation, and Control Conference, 1-14, Toronto, Ontario Canada.

2. Bernhard, P. (2002), Survey of Linear Quadratic Robust Control, Macroeconomic Dynamics, 6, 19-39.

3. Bousson, K., Velosa, C.M.N. (2014), Robust Control and Synchronization of Chaotic Systems with Actuator Constraints, P. Vasant (Ed.), Handbook of Research on Artificial Intelligence Techniques and Algorithms, 1-43, IGI Global.

4. Chen, C.L., Peng, C.C., Yau, H.T. (2012), High-order Sliding Mode Controller with Backstepping Design for Aeroelastic Systems, Communications in Nonlinear Science and Numerical Simulation, 17(4), 1813-1823.

5. Demenkov, M. (2008), Structural Instability Induced by Actuator Constraints in Controlled Aeroelastic System. In Proceedings of the 6th EUROMECH Nonlinear Dynamics Conference (ENOC 2008). Saint Petersburg, Russia.

6. Deng, L., Xu, J. (2010), Control and Synchronization for Uncertain Chaotic Systems with LMI Approach, Chinese Control and Decision Conference (CCDC), 1695-1700.

7. Galeani, S., Teel, A.R., Zaccarian, L. (2007), Constructive Nonlinear Anti-windup Design for Exponentially Unstable Linear Plants, Systems \& Control Letters, 56(5), 357-365.

8. Hippe, P. (2006), Windup In Control: Its Effects and Their Prevention, Springer.

9. Horowitz, I. (2001), Survey of Quantitative Feedback Theory (QFT) International Journal of Robust and Nonlinear Control, 11(10), 887921.

10. Huang, J. (2004), Nonlinear Output Regulation: Theory and Applications, Philadelphia: Siam.

11. Ott, E., Grebogi, C., Yorke, J.A. (1990), Controlling Chaos, Physical Review Letters, 64(11), 1196-1199.

12. Pecora, L.M., Carroll, T.L. (1990), Synchronization in Chaotic Systems, Physical Review Letters, 64(8), 821-824.

13. Saberi, A., Stoorvogel, A.A., Sannuti, P. (2011), Control of Linear Systems with Regulation and Input Constraints, Springer; Reprint of the original 1st ed.

14. Tanaka, K., Wang, H. O. (2001), Fuzzy Control Systems Design and Analysis: A Linear Matrix Inequality Approach (1st ed.), WileyInterscience.

15. Velosa, C.M.N., Bousson, K. (2014), Suppression of Chaotic Modes in Spacecraft with Asymmetric Actuator Constraints, Submitted to the Proceedings of the Institution of Mechanical Engineers, Part G: Journal of Aerospace Engineering.

16. Velosa, C. M. N., Bousson, K. (2015), Synchronization of Chaotic Systems with Bounded Controls, International Review of Automatic Control (IREACO), (To appear). 
17. Vikhorev, K. S., Goman, M. G., Demenkov, M. N. (2008), Effect of Control Constraints on Active Stabilization of Flutter Instability, Proceedings of The Seventh International Conference on Mathematical Problems in Engineering Aerospace and Sciences (ICNPAA 2008), 1-8, Genoa, Italy.

18. Wang, C.-C., Chen, C.-L., Yau, H.-T. (2013), Bifurcation and Chaotic Analysis of Aeroelastic Systems, Journal of Computational and Nonlinear Dynamics, 9(2), 021004 (13 pages).

19. Zames, G. (1981), Feedback and Optimal Sensitivity: Model Reference Transformations, Multiplicative Seminorms, and Approximate Inverses, IEEE Transactions on Automatic Control, 26(2), 301-320.

Acknowledgments: This research was conducted in the Aeronautics and Astronautics Research Unit of the University of Beira Interior (LAETA UBI / AeroG) at Covilhã, Portugal, and supported by the Portuguese Foundation for Science and Technology (FCT).

Nomenclature: $V$ - freestream velocity; $h$ - plunge displacement; $\alpha$ - angle of attack / pitch angle; $\gamma$ - leading-edge control surface deflection angle; $\beta$ - trailing-edge control surface deflection angle; $\rho$ - air density; $a$ - dimensionless dist. from mid-chord to elastic axis position; $b$ - wing section half-chord; $s$ - wing section span; $k_{h}$ - plunge displacement stiffness coefficient; $k_{\alpha}$ - pitch spring stiffness coefficient; $c_{h}$ - plunge displacement damping coefficient; $c_{\alpha}$ - pitch angle damping coefficient; $m_{\text {wing }}$ - mass of wing section; $m_{W}$ - total wing section plus mount mass; $m_{T}-$ total mass of pitch-plunge system; $I_{\text {cam }}-$ pitch cam moment of inertia; $I_{c g \text {-wing }}$ - wing section moment of inertia about the center of gravity; $I_{\alpha}$ - moment of inertia; $x_{\alpha}$ - dimensionless distance between the center of gravity and the neutral axis; $C_{l \alpha}$ - lift coefficient per unit of angle of attack, $\partial l / \partial \alpha ; C_{m \alpha}-$ moment coefficient per unit of angle of attack, $\partial m / \partial \alpha ; C_{l \beta}$ - lift coefficient per unit of wing trailingedge control surface deflection, $\partial l / \partial \beta ; C_{m \beta}-$ moment coefficient per unit of wing trailing-edge control surface deflection, $\partial m / \partial \beta ; C_{l \gamma}-$ lift coefficient per unit of wing leading-edge control surface deflection, $\partial l / \partial \gamma ; C_{m \gamma}-$ moment coefficient per unit of wing leading-edge control surface deflection, $\partial m / \partial \gamma ; C_{m \alpha-e f f}$ - effective control moment derivative due to angle of attack; $C_{m \beta-e f f}$ - effective control moment derivative due to trailing-edge control surface deflection; $C_{m \gamma-e f f}-$ effective control moment derivative due to leading-edge control surface deflection.
Appendix 1: Solving the Regulator Equations

The following system, whose the first equation is a Sylvester equation, is known as the linear regulator equations (or also as the Francis' equations), and its resolution is essential to determine the control law presented in this paper.

$\Pi S=A \Pi+B_{1} \Gamma+B_{2}$

$0=C_{1} \Pi+D_{12}$

Let vec: $\mathbb{R}^{l \times c} \rightarrow \mathbb{R}^{l c \times 1}$ be a vector-valued function of a matrix such that, for any $X \in \mathbb{R}^{l \times c}$ :

$\operatorname{vec}(X)=\left[\begin{array}{c}X_{1} \\ \vdots \\ X_{c}\end{array}\right]$

where, for $i=1, \ldots, c, X_{i}$ is the $i$ th column of $X$. Applying this transformation in all terms of the system yields:

$\operatorname{vec}(\Pi S)-\operatorname{vec}(A \Pi)-\operatorname{vec}\left(B_{1} \Gamma\right)=\operatorname{vec}\left(B_{2}\right)$

$\operatorname{vec}\left(C_{1} \Pi\right)=\operatorname{vec}\left(-D_{12}\right)$

Since $A \in \mathbb{R}^{n \times n}, \quad B \in \mathbb{R}^{n \times m}, \quad S \in \mathbb{R}^{s \times s}, \quad B_{2} \in \mathbb{R}^{n \times s}, \quad C_{1} \in$ $\mathbb{R}^{p \times n}$, matrices $\Pi$ and $\Gamma$ have dimensions $n \times s$ and $m \times s$ respectively. Through some prepositions which can be found in (Huang, 2004), equations above can be rewritten as:

$\left(S^{T} \otimes I_{n}\right) \operatorname{vec}(\Pi)-\left(I_{s} \otimes A\right) \operatorname{vec}(\Pi)-\left(I_{s} \otimes B_{1}\right) \operatorname{vec}(\Gamma)=\operatorname{vec}\left(B_{2}\right)$

$\left(I_{s} \otimes C_{1}\right) \operatorname{vec}(\Pi)=\operatorname{vec}\left(-D_{12}\right)$

where $I_{S} \otimes A$ denotes the Kronecker product between matrices $I_{S}$ and $A$. Through the following mathematical manipulation:

$$
\begin{aligned}
& \overbrace{\left(S^{T} \otimes I_{n}-I_{S} \otimes A\right)}^{A_{*}} \operatorname{vec}(\Pi)-\overbrace{\left(I_{S} \otimes B\right)}^{B_{*}} \operatorname{vec}(\Gamma)=\operatorname{vec}\left(B_{2}\right) \\
& \underbrace{\left(I_{S} \otimes C_{1}\right)}_{C_{*}} \operatorname{vec}(\Pi)=\operatorname{vec}\left(-D_{12}\right)
\end{aligned}
$$

these equations can be transformed into a standard linear algebraic system of the form:

$\underbrace{\left[\begin{array}{cc}A_{*} & -B_{*} \\ C_{*} & 0_{s p \times s m}\end{array}\right]}_{H} \underbrace{\left[\begin{array}{c}\operatorname{vec}(\Pi) \\ \operatorname{vec}(\Gamma)\end{array}\right]}_{z}=\underbrace{\left[\begin{array}{c}\operatorname{vec}\left(B_{2}\right) \\ \operatorname{vec}\left(-D_{12}\right)\end{array}\right]}_{b}$

and matrices $\Pi$ and $\Gamma$ calculated as $z=H^{-1} b$.

\section{Appendix 2: Solving the $H_{\infty}$-like Riccati Equation}

The continuous-time algebraic Riccati equation (14), transcribed below for the sake of convenience:

$A^{T} P+P A-P B_{1} B_{1}^{T} P+\gamma^{-2} P B_{3} B_{3}^{T} P+Q=0$

can be rewritten, after a mathematical manipulation, as:

$A^{T} P+P A-P \underbrace{\left[\begin{array}{ll}B_{3} & B_{1}\end{array}\right]}_{B} \underbrace{\left[\begin{array}{cc}-\gamma^{2} I_{n+q} & 0_{(n+q) \times m} \\ 0_{m \times(n+q)} & I_{m}\end{array}\right]^{-1}}_{R} \underbrace{\left[\begin{array}{c}B_{3}^{T} \\ B_{1}^{T}\end{array}\right]}_{B^{T}} P+Q=0$

where $I$ and 0 denote respectively identity and zero matrices.

Its unique solution, $P \geq 0, P=P^{T}$, can be easily computed, if there exists, by the following function provided in Matlab: $P=\operatorname{care}(A, B, Q, R)$. 